\title{
OPEN DISCUSSION
}

\author{
Chairman of the morning session: A. R. Hyland \\ Chairman of the afternoon session: R. I. Thompson
}

Thompson: I have persuaded Dr Townes to say a few words about the infrared work he is doing. He has been mainly concerned with a project involving spatial interferometry in the infrared. However, the technique does have some applications in infrared spectroscopy.

Townes: Let us talk about high spectral resolutions work using heterodyne spectroscopy. Of course that kind of spectroscopy has been used in the radio region a long time and obviously gives immediately almost infinite resolution because one simply needs to mix with a local oscillator the incoming signal and then filter out the resulting signal with various radio frequencies filters which one can make as narrow as he pleases. The problem is not the resolution then but poor signal to noise. A heterodyne detector has basically a noise present due to the uncertainty principle. One can get rid of a noise temperature of $h v / k$ when $v$ is the frequency and $k$ is the Boltzmann constant. This means that at $10 \mu$ for example, the noise temperature of the detector is necessarily high. It is about $15000 \mathrm{deg}$. And so one is looking at a background of $15000 \mathrm{deg}$ and trying to determine some stellar spectrum against it. Now that is not impossible, but it does mean that about $10 \mu$ is as high a frequency as heterodyne techniques are likely to be useful. I think, one might go another factor of two or so. Of course if we go down to the radio region then this noise temperature is of the order of one degree or so. I have given you only the theoretical limit. In fact our present detectors miss that by about one order of magnitude. Detectors in the laboratory have done better and miss that theoretical limit by maybe a factor of three only, and certainly one can get such detectors in the field. (This is the theoretical result I say one order of magnitude.) Actually there are two kinds of detector, a photoconductor has a noise temperature twice that, and we miss that by a factor of 10 so we have $20 h v / k$, and in the lab this has gotten down to maybe about three or four times $h v / k$ and that certainly can be achieved in the field. The result is that when we look at a star we can get a ten to one signal of noise in the band width which we have which is $13000 \mathrm{mHz}$ and get ten to one signal to noise in something like five to ten minutes for the brighter stars. This means that one can divide that band width up then with filters into practically maybe 10 or 20 units and with reasonable observation times can get a signal. We are using a 30-in. telescope. Obviously a 100-in. telescope would give you 10 times more signal to noise, so that for the brighter stars this a perfectly practical kind of technique. One might ask do you need that resolution because most spectral lines in the $10 \mu$ region are going to be as broad as $1 / 20$ of a wave number probably or something like that, so that most work can be done with other kinds of interferometry. On the other hand in cool stellar shells there is a need at least there for higher resolu- 
tion. We also hope to use this for planetary work for the planetary atmospheric lines where high resolution is needed. And so if one wants a really high resolution this is a way of doing it. On Mars, for example, we get a signal to noise of 10 in about five seconds. Mars because of its broader solid angle is much more favourable than stars and also a good deal cooler, so that in the atmosphere of Mars one can look at $\mathrm{CO}_{2}$ lines in enormous detail getting $1 / 1000$ of a wave number easily, if that's what resolution is needed, and it is just about needed down to that level for some of these cases. For stars the time required is longer but for the brighter stars one certainly can detect a spectrum. This study has been carried about by two students Betts and Jansen aided to a lesser extent by two other students, Gailhouse and Petersen, and Petersen, Betts and Jansen in particular hope to look at some spectrum of Mars before long. We have been headed in a different direction and have not really produced any spectra yet but they hope to do that before long. Now I must warn you of another technical difficulty. This is a difficult technical field. Now we can get this resolution you look at one time at a band width of maybe $15000 \mathrm{mHz}$, but that is $1 / 20$ of a wave number. So your total field of view is $1 / 20$ of a wave number, and you can break that up as finely as you wish. Now, you say as in the radio frequency range let us tune our local oscillator around and we move this $1 / 20$ of a wave number around, and we can then get a complete spectrum and look in enormous detail at any reasonable spectral region we wish. The trouble with that is that the local oscillators and the infrared lasers are not all that tunable and flexible. You will have heard of tunable solid state lasers in this region, and they work, but they do not work consistently at the power levels required for the presently available detectors. They do in certain laboratories and for certain limited times work at power levels adequate for certain special detectors, and none of these things are commercially available, but within the course of perhaps two or three years they ought to be commercially available, and sooner than that on a personalized kind of basis. With that one can then tune through the spectrum, tune the local oscillator getting this exceedingly high resolution obviously needed for only very special problems, but they are undoubtedly giving us a great deal of information. You may possibly have seen such spectra taken in the laboratory where the problem is easy. In the laboratory from an absorption cell you can have a tunable local oscillator, a tunable laser, simply a receiver on the other end, and then the resolution, not by heterodyne techniques, but simply because of the high spectral purity of the laser, is just fantastic. And people get down to resolutions of anything you want but normally down to about $1 / 30000$ of a wave number. With nice heavy gases with lots of rotational structures this is sometimes needed.

Schwarzschild: I would certainly like to emphasize how dreadfully important this type of work is for any stellar evolution in the advanced phases. Practically everything interesting happens exactly in the stars that you have been discussing all day. I am a little more squeamish being a theoretician of the use of our theoretical work that some of you are doing, because I think it is less certain than you think, but that is a good state of affairs. Obviously in spite of my very great admiration of the work that you are attempting there are additional points that $I$ hope by and by you will come to. 
One of the points, for example, I will suspect will come home to you and to us is the question does one have to worry already in the photosphere when one is building stellar models as some of you have been doing about the formation of grains adding to the opacity or vice versa. Can one in this range, which is not the range in which the grains radiate by themselves, possibly detect the existence of a more or less neutral absorber? I mean that for constructing for using your model atmosphere as boundary conditions for our stellar interiors will become important.

However, I would like to come back once more and emphasize anything you can do to learn about the hydrodynamics of the atmospheres in addition of course to the abundances is of fundamental importance to the interior.

Thompson: I think before this session draws to a close we ought to give a very hearty round of applause for Dr Fujita for working so hard to bring this together (applause). 